\title{
DUBLIZIEREN UND LESEN IN DEN ZEITEN DER PANDEMIE
}

\section{von Jürgen Stickelberger und Max Vögler}

Zusammenfassung: Wie hat sich die Disseminierung und das Rezipieren wissenschaftlicher Ergebnisse während der Pandemie geändert? In diesem Beitrag berichten die Autoren über die Erfahrungen des Wissenschaftsverlages Elsevier im „Covid-Jahr 2020". Wie waren aus ihrer Sicht die Wissenschaft und das Verlagswesen betroffen? Die Autoren werden von der Flut an Covid-Artikeln berichten, sowie auch erzählen, wie ihre eigene Arbeit betroffen war. Anschließend wird die Literaturversorgung in Österreich während der Pandemie untersucht: was können wir über das veränderte Nutzer*innenverhalten in den letzten 9 Monaten lernen?

Schlagwörter: Elsevier; COVID-19; Publikationswesen; Nutzungsverhalten

\section{PUBLISHING AND READING IN THE TIMES OF THE PANDEMIC}

Abstract: How did dissemination and reception of scientific results change during the pandemic? In this article, the authors report on the experiences of the scientific publisher Elsevier in the "Covid Year 2020". From their perspective, how were science and publishing affected? The authors will tell about the flood of COVID articles, as well as how their own work was affected. Afterwards, the literature supply in Austria during the pandemic will be explored: what can we learn about the changing user behaviour during the nine months?

Keywords: Elsevier; COVID-19; publication system; user behaviour

DOI: https://doi.org/10.31263/voebm.v73i3-4.5554

(C) Jürgen Stickelberger, Max Vögler

Dieses Werk ist - exkl. einzelner Logos und Abbildungen - lizenziert unter einer Creative-Commons-Lizenz Namensnennung 4.0 International-Lizenz 


\section{Einleitung}

Die COVID-19-Pandemie hat unser aller Leben nachhaltig verändert - die wissenschaftliche Arbeit ist davon nicht ausgenommen. Einerseits wird unsere Arbeit entschleunigt: Viele von uns, deren Kalender oft schon Monate im Voraus mit verschiedenen Konferenzen belegt waren, fanden sich nun täglich in den eigenen vier Wänden wieder. Neben der Arbeit spielt plötzlich bei einigen beispielsweise die Kinderbetreuung eine große Rolle. Auf die anfänglich empfundene „Schockstarre“ folgte aber schnell ein zielorientiertes Umdenken. So haben die verschiedenen Disziplinen ihren ganz eigenen Umgang gefunden: Von Videokonferenzen über Social Media, bis hin zu Preprint-Kommentaren. Insgesamt kann man sagen, dass die Digitalisierung der informellen Kommunikation einer der interessantesten Aspekte der Pandemie ist.

Andererseits sehen wir jedoch auch eine signifikante Beschleunigung in der Disseminierung wissenschaftlicher Inhalte, die durch die COVID-19-Pandemie aus der Not heraus nahezu rasant geworden ist: Nachdem das Virus im Dezember 2019 erstmalig in Wuhan, China, als Auslöser von COVID-19 identifiziert wurde, lagen schon Ende Januar 2020 erste wissenschaftliche Ergebnisse zu Virus und Erkrankung in The Lancet vor, begleitet von einem Aufruf zum Daten-Sharing. Nur einen Monat später publizierten chinesische Wissenschaftler*innen die genomische Sequenz des Virus. Und dann überschlugen sich die Ereignisse nahezu: Seit Anfang des Jahres hat allein der Wissenschaftsverlag Elsevier über 30.000 Artikel, Kommentare, Reviews und weitere Beiträge zu COVID-19 veröffentlicht, insgesamt existieren ca. 84.000 wissenschaftliche Publikationen - eine noch nie dagewesene globale Forschungsintensität mit einer Springflut an Ergebnissen und Erkenntnissen zu einem einzigen Thema. ${ }^{1}$ Auch allgemein ist die Zahl der bei Elsevier-Journalen eingereichten Manuskripte von Januar bis Oktober 2020 mit 58\% signifikant gestiegen. Der zu verzeichnende Zuwachs war am höchsten in den Gesundheitswissenschaften, wo der Anstieg im Vergleich zum Vorjahr sogar $92 \%$ betrug. ${ }^{2}$ Dieser Trend war auch bei Autor*innen in Österreich zu beobachten: von Januar bis Oktober 2020 betrug der Anstieg an bei Elsevier eingereichten Artikeln im Vergleich zum Vorjahreszeitraum 21,4\%. Im Jahr 2019 konnten wir lediglich eine Zunahme von 1,84\% im Vergleich zum Vorjahreszeitraum messen. Dieser Zuwachs hat nicht nur zu einer enormen Arbeitsbelastung für die Verlagsmitarbeiter*innen sowie Editor*innen der einzelnen Fachzeitschriften, sondern auch zu einem enormen Mehraufwand für das wissenschaftliche Begutachtungssystem geführt.

Der großen Aktualität und dem Willen zum raschen Erkenntnisfortschritt geschuldet, erscheint die überwiegende Mehrheit der einschlägigen 
Publikationen als Preprint - ohne das Gütesiegel eines Peer-Review-Prozesses. So sinnvoll und akzeptabel eine solche Strategie in Notzeiten sein kann, so wichtig ist es doch für Verlage, die entsprechenden Artikeltypen vor diesem Hintergrund entsprechend zu kennzeichnen. So haben wir mit dem Wachstum im Preprint-Bereich auch bei den Journals viel Arbeit gesehen, um die unterschiedlichen Beitragsarten für die deutlich breitere Öffentlichkeit besser zu kennzeichnen, beispielsweise in The Lancet. ${ }^{3}$

Auch in der Nutzung der von uns angebotenen Lösungen konnten wir signifikante Veränderungen feststellen. In Österreich lag die Abdeckung mit Remote Access wie Shibboleth, VPN und anderen Lösungen auf sehr hohem Niveau. Es waren praktisch keine Zugangsbeschränkungen zu beobachten. Darüber hinaus haben wir weitere Lösungen geschaffen, um gemeinsam gegen die akute Situation zu reagieren und darüber hinaus sicherzustellen, dass die Nutzer*innen versorgt werden.

Im ersten Schritt wurde das Novel Coronavirus Information Center ${ }^{4}$ mit dem Ziel ins Leben gerufen, der Wissenschaftscommunity, dem klinischen Personal sowie Patient*innen relevante Literatur und Forschungsdaten zu dem neuartigen Virus zur Verfügung zu stellen. Besonderes Augenmerk lag darauf, Forscher*innen die Daten an die Hand zu geben, die sie benötigen, um an Impfstoffen forschen zu können. Ende Oktober verzeichneten wir über 150 Mio. Downloads. Hier hat sich Elsevier dem Aufruf der wichtigsten Wissenschaftsberater*innen weltweit angeschlossen, für die Dauer der Krise relevante Publikationen und Daten verfügbar zu machen. ${ }^{5}$

Weiterhin stellte Elsevier Zugang zu weiteren relevanten Produkten für Forscher*innen, die an Lösungen rundum COVID-19 arbeiten, zur Verfügung. Dazu gehören beispielsweise Embase, Clinical Key, und Pure, ein Portal, über das Forscher*innen mögliche Kollaborationspartner*innen identifizieren können. ${ }^{6}$ Seitens der Kolleg* innen aus dem D-A-CH-Team wurden Webinare angeboten, um über die verschiedenen Remote-Access-Optionen aufzuklären. Auch darüber hinaus wurden weltweit ca. 250 Lehrbücher Einrichtungen kostenlos zur Verfügung gestellt. In Österreich wurde dieses Angebot dankend angenommen und für die Einrichtungen in Österreich um weitere Zugangsservices ergänzt. Ende August erfolgte die Abschaltung des Zugangs.

\section{Wie hat die Pandemie das Arbeiten der Mitarbeiter*innen beeinflusst?}

Für den Arbeitsalltag bei Elsevier brachte die Pandemie ebenfalls signifikante Umstellungen mit sich: Sowohl für Publisher und Editor*innen als auch für die Kolleg*innen im Vertrieb und in der Entwicklung bei Elsevier wurden Dienstreisen und Veranstaltungen zeitnah abgesagt. Das betraf 
vor allem eine für Anfang Februar geplante große interne Konferenz sowie weiterhin alle externen Veranstaltungen, die sukzessive gestrichen wurden. Bei den Editorial Boards gab es positive Nebeneffekte: Beispielsweise trafen sich Editorial Boards, die sich bislang oft nur einmal im Jahr auf einer Fachkonferenz getroffen hatten, nun regelmäßiger, da für fast alle Wissenschaftler*innen auf der Welt Videokonferenztechnologie zum neuen Standard wurde. Europa kann hier als kleiner "Zeitzonen-Gewinner" gesehen werden, da sich 13:00 Uhr CET vielfach als Kompromiss für internationale Konferenzen und Meetings etablierte.

Mit Beginn des Jahres 2021 sind Videokonferenzen der Regelfall geworden. Besonders bemerkenswert ist dabei, dass durch die Einsparung der Zeit, die sonst für An- und Abreise zu Konferenzen eingeplant werden musste, ein enormer Effizienzgewinn zu verzeichnen war. Dem entgegen steht jedoch ein Verlust des informellen Austauschs, welcher bislang im Rahmen von Konferenzen stattgefunden hatte. Auch wenn der persönliche Kontakt unter Nutzung entsprechender Videokonferenzdienste aufrecht erhalten werden konnte, insbesondere, wenn sowohl Video- als auch Audioübertragung gewählt wurden, so entfielen die oft fruchtbaren Diskussionen am Rande von Tagungen. Diese Anlässe hatten in der Vergangenheit oft willkommene Anknüpfungspunkte geboten, die durch die Pandemie leider entfielen. Daraus entstand jedoch auch ein neues Bewusstsein für die Kontaktpflege.

\section{Die Nutzung und das Nutzungsverhalten}

Die folgenden Graphiken sollen die Nutzung und das Nutzungsverhalten besser veranschaulichen:

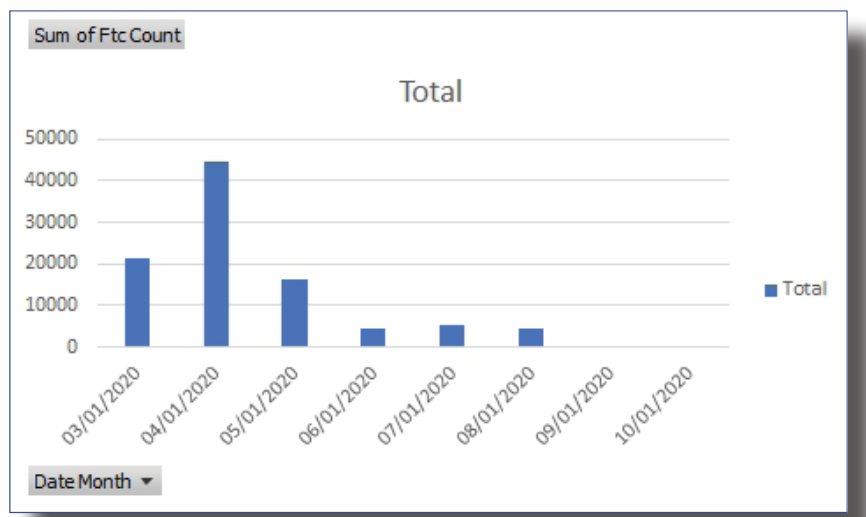

Abb. 1: Nutzung nach Kapiteln während des Zugangs zum COVID-Trial

Mitteilungen der VÖB 73 (2020) Nr. 3/4 
Der Vergleich zu den Niederlanden, Schweden und Norwegen, die mit dem demographischen Profil und ähnlich großer Landschaft an Universitäten und Hochschulen verglichen werden können, zeigt die hohe Nutzung an den österreichischen Einrichtungen.

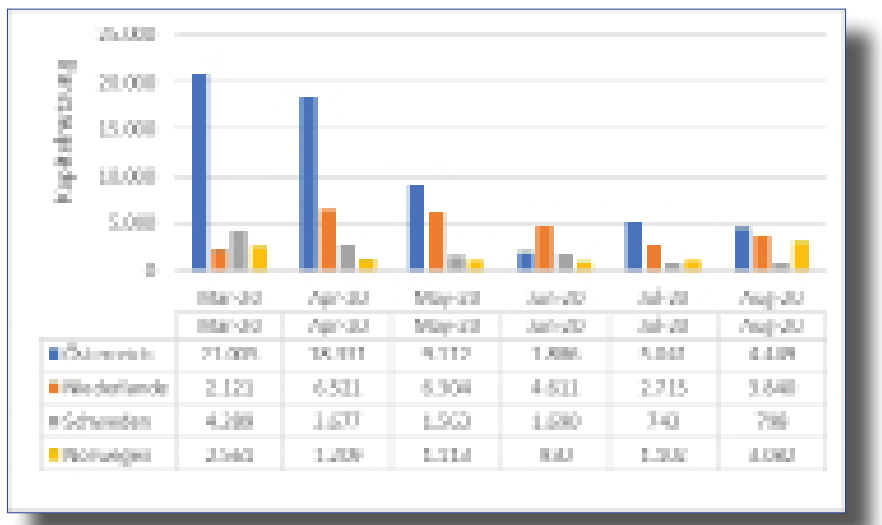

Abb. 2: Gesamtnutzung nach Kapitel im Vergleich zum COVID-Trial

Die Nutzung der in Österreich lizenzierten Journale auf ScienceDirect zeigt, dass das Vorjahresniveau bereits im November erreicht wurde.

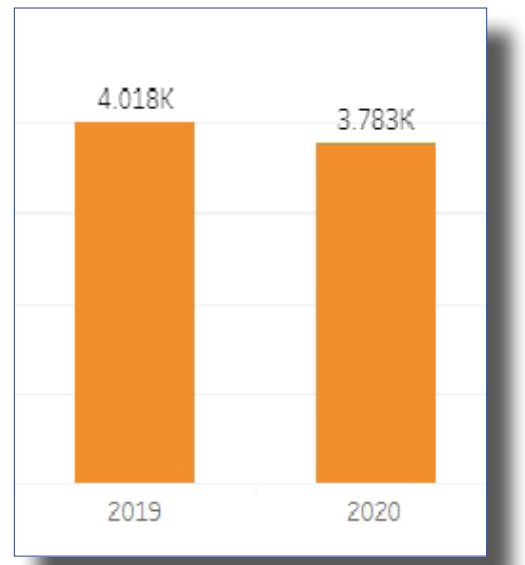

Abb. 3: Nutzung nach Item Requests Gesamtjahr 2019 vs. Januar bis Oktober 2020 an den akademischen Einrichtungen (Volltextnutzung in 1.000)

Dabei ist die Datengrundlage jeweils die Nutzung aus den akademischen Einrichtungen in Österreich herangezogen worden. 


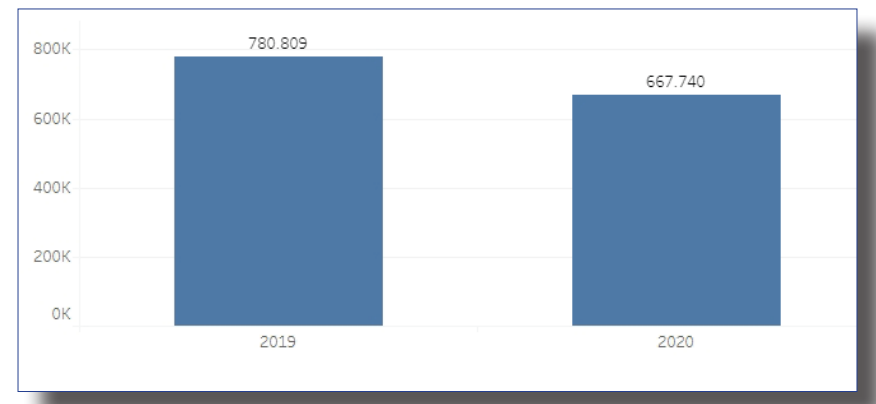

Abb. 4: Suchanfragen auf ScienceDirect Gesamtjahr 2019 vs. Januar bis Oktober 2020 an den akademischen Einrichtungen

Die öffentliche Wahrnehmung sowie Nutzungsstatistiken von ScienceDirect zeigt uns die große Relevanz wissenschaftlicher Inhalte, die in qualitätsgesicherten Journals in Zeiten einer Pandemie erscheinen.

\begin{tabular}{|c|c|c|c|c|c|}
\hline \multirow[b]{2}{*}{$\square$} & \multirow[b]{2}{*}{ Sopous Source } & \multicolumn{2}{|l|}{ Scholaty } & \multicolumn{2}{|l|}{ Field:Weighied } \\
\hline & & Output & vemencount $\checkmark$ & Ctasion Impat $\checkmark \downarrow$ & Citxion Coum $\checkmark$ \\
\hline 110 & New England jouresal of Medicine & 333 & 7,499 & 48.45 & 33,765 \\
\hline H口 & The Lancet & $\$ \%$ & 22,520 & 11.99 & 44,651 \\
\hline$\# \square$ & The Laneet Respiratery Med oine & 133 & 2,830 & 33.47 & 7,924 \\
\hline 10 & Oinieal l-fostieus Divases & 98 & 2.143 & 31.40 & 7.607 \\
\hline 10 & Jouras of Thrembotis and Haemostasis & ss & 92 & 28.97 & 5,202 \\
\hline 10 & JaMA- Joumbl of the Americas Med eal Aasceiztion & 391 & 6,276 & 27.13 & 27,240 \\
\hline 10 & Noture & 294 & 5,875 & 26.46 & 11,267 \\
\hline 110 & Noture Madisne & 132 & 2,246 & 21.33 & 5,030 \\
\hline \#प & Emerging Microbes and infections & 108 & 6.400 & 19.82 & 3,690 \\
\hline $1 \square$ & Sevence & $\mathrm{k} 22$ & 4,907 & 18.95 & 9.260 \\
\hline ED & Eurosureillance & 126 & 3,541 & 18.18 & 4,096 \\
\hline $1 \square$ & Intenine Care Madibiate & 125 & 1,560 & 13.05 & 4,866 \\
\hline $\mathrm{HO}$ & The Lancet Pypchiaty & 93 & 3,195 & 16.90 & 3,034 \\
\hline
\end{tabular}

Abb. 5: Auflistung der wichtigsten Fachzeitschriften mit COVID-19 Forschung, gelistet nach Field-Weighted Citation Impact (FWCI). (Quelle: www.scival.com, abgerufen am 2. Dezember 2020)

\section{Was haben wir aus dieser Entwicklung gelernt?}

Die konsequente Implementierung und Weiterentwicklung von EnterpriseResource-Planning-Systemen, die der Verwaltung von Geschäftsabläufen 
und deren Prozessen dienen, in die Cloud ist ein essentieller Schritt, um einen ungestörten Betriebsablauf sicherzustellen. Mit solchen Cloud-Lösungen wurde eine nahtlose Umstellung auf die Arbeit aus dem Homeoffice bereits im Vorfeld sichergestellt und somit unternehmensweit die erforderlichen Systeme zum Beginn des Lockdown einsatzbereit. Ohne leistungsfähige Anbindung an diese Webservices wäre der Betriebsablauf erheblich beeinträchtigt gewesen.

Die COVID-19-Pandemie hat unser Arbeitsleben verändert: Von der Art, wie wir miteinander kommunizieren bis hin zur Art und Geschwindigkeit der Disseminierung wissenschaftlicher Arbeiten. Angesichts der Vielzahl von Einreichungen und Publikationen in verschiedenen Formaten wird es in Zukunft noch wichtiger, transparent zu kommunizieren, ob es sich beispielsweise um Preprints oder Artikel handelt, die bereits den Peer-Review-Prozess durchlaufen haben. Tatsächlich kam der Wissenschaftskommunikation während der Pandemie eine tragende Rolle zu. Eine der wichtigsten Erkenntnisse, die wir aus der Pandemie mitnehmen, ist daher auch, dass es nicht genug ist, wissenschaftliches Wissen zu produzieren, um eine effiziente wissenschaftliche Versorgung von Politik und Gesellschaft zu gewährleisten. Wissenschaftler*innen, Fachgesellschaften und nationale Akademien, Wissenschaftsjournalist*innen sowie die Wissenschaftsverlage müssen gemeinsam agieren. Nur so können wir auch in Zukunft sicherstellen, dass wissenschaftliches Wissen seinen berechtigten Beitrag zu sozialen Debatten und gesellschaftlichen Herausforderungen leisten kann.

Jürgen Stickelberger

Elsevier

E-Mail: j.stickelberger@elsevier.com

Dr. Max Vögler ORCID iD: https://orcid.org/0000-0003-4560-5145

Elsevier E-Mail: m.voegler@elsevier.com 
* Alle Websites und Links wurden zuletzt am 15. Januar 2021 aufgerufen.

1 Vgl. John P.A. Ioannidis, Maia Salholz-Hillel, Kevin W. Boyack, Jeroen Baas (2020). The rapid, massive infection of the scientific literature and authors by COVID-19. bioRxiv, 2020.12.15.422900. https:// doi.org/10.1101/2020.12.15.422900 [Preprint]. Die durch Elsevier veröffentlichten Publikationen können auf ScienceDirect abgerufen werden: https://www.sciencedirect.com/articlelist/covid. Für eine Gesamtanalyse ist das Coronavirus Research Repository von 1science sehr nützlich: https://coronavirus.1science.com/search. Vgl. auch Kent Anderson (2021). Covid-19 Preprint Counts Are Inflated. The Geyser (4 January). https://thegeyser.substack.com/p/covid-19-preprint-countsare-inflated

2 Christos Petrou (2020). Scientific output in the year of COVID. Scholarly Kitchen (19 November). https://scholarlykitchen.sspnet. org/2020/11/19/guest-post-scientific-output-in-the-year-of-covid/; Flaminio Squazzoni, Giangiacomo Bravo, Francisco Grimaldo, Daniel Garcia-Costa, Mike Farjam, Bahar Mehmani (2020). No Tickets for Women in the COVID-19 Race? A Study on Manuscript Submissions and Reviews in 2347 Elsevier Journals during the Pandemic. SSRN, 3712813. http://dx.doi.org/10.2139/ssrn.3712813 [Preprint].

3 Vgl. The Lancet (2020). The Lancet: What we Publish and Why. YouTube. https://youtu.be/xvxugXJW4eA

4 Elsevier, Novel Coronavirus Information Center: https://www.elsevier. com/connect/coronavirus-information-center

$5 \mathrm{Vgl}$. https://ec.europa.eu/info/sites/info/files/research_and_innovation/news/documents/coronavirus_open_access_letter.pdf

6 Elsevier, Coronavirus Research Hub: https://www.elsevier.com/clinicalsolutions/coronavirus-research-hub. Für das Pure Portal vgl. https:// covid19.elsevierpure.com/ 